\title{
Correction to: Causes, background, and characteristics of binocular diplopia in the elderly
}

\author{
Manami Kawai $^{1} \cdot$ Toshiaki Goseki $^{1,2} \cdot$ Hitoshi Ishikawa ${ }^{1,3} \cdot$ Miki Hoshina $^{4} \cdot$ Nobuyuki Shoji $^{1,2}$
}

Published online: 2 July 2019

(c) Japanese Ophthalmological Society 2019

\section{Correction to: \\ Japanese Journal of Ophthalmology (2018) 62:659-666 https://doi.org/10.1007/s10384-018-0617-2}

The article 'Causes, background, and characteristics of binocular diplopia in the elderly' by Manami Kawai, Toshiaki Goseki, Hitoshi Ishikawa, Miki Hoshina, Nobuyuki Shoji was published by the Japanese Journal of Ophthalmology in 2018 (Vol. 62, pp. 659-666 https://doi.org/10.1007/ s10384-018-0617-2). Dr. Goseki subsequently informed the editors of this journal that the expression: divergence insufficiency exotropia (page 662, right column, line 14) is incorrect. Specifically, the sentence should read: convergence insufficiency exotropia.

Publisher's Note Springer Nature remains neutral with regard to jurisdictional claims in published maps and institutional affiliations.

The original article can be found online at https://doi.org/10.1007/ s10384-018-0617-2.

Toshiaki Goseki gosekikun@aol.com

1 Graduate School of Medical Sciences, Kitasato University, Sagamihara, Kanagawa, Japan

2 Department of Ophthalmology, Kitasato University, 1-15-1, Kitasato, Minami-ku, Sagamihara, Kanagawa 252-0374, Japan

3 School of Allied Health Sciences, Kitasato University, Sagamihara, Kanagawa, Japan

4 Sanno Hospital, Minato-ku, Tokyo, Japan 